\title{
Electric Field Distribution Analysis of Blood Cancer as a Potential Blood Cancer Therapy
}

\author{
Miftakhul Firdhaus, Ulya Farahdina, Vinda Zakiyatuz Zulfa, Endarko, \\ Agus Rubiyanto, Nasori ${ }^{*}$ \\ Laboratory of Medical Physics and Biophysics, Departement of Physics, \\ Institut Teknologi Sepuluh Nopember, Kampus ITS - Surabaya 60111, \\ East Java, Indonesia \\ *E-mail: kanganas01@gmail.com
}

\begin{abstract}
Blood cancer causes a significant increase in the concentration of Leukocytes, which can be broken down through dielectrophoresis and electrochemical procedures. Therefore, the electric field plays an important role in the migration of leukocytes to high voltage areas. This is because different electrode arrangements produce varying electric field distributions. Furthermore, this study applied finite element methods to generate electric fields when electrodes with an AC voltage were applied to blood placed in a chamber. Therefore, in this study, variations of mediums and electrode arrangements were investigated, which led to the recommendation of 3 models. The objective was to investigate electrode arrangements that produce optimal electric field distribution for the three models to exhibit a booster of electric field distribution. The maximum electric field is generated close to the electrode $(Z=2 \mathrm{~mm}$ and $\mathrm{Z}=92 \mathrm{~mm}$ ) for any material (i.e. normal blood, $B$ lymphocyte, and T lymphocyte) with values of $22.6 \mathrm{~V} / \mathrm{m}$ and $23.47 \mathrm{~V} / \mathrm{m}, 22.85 \mathrm{~V} / \mathrm{m}$ and 22.97 $\mathrm{V} / \mathrm{m}$, and $24.88 \mathrm{~V} / \mathrm{m}$ and $25.01 \mathrm{~V} / \mathrm{m}$. Based on principle, lymphocytes in the blood result in positive dielectrophoresis, since they migrate to a higher electric field close to the electrode, with enough input voltage to turn the electrochemical process on the leukocytes into electric current. Furthermore, this study provides new perspectives and ideas, which have not been revealed in previous studies on blood cancer therapy using the electric field of Ag electrode in blood cancer distribution.
\end{abstract}

Keywords: blood cancer, dielectrophoresis, electric field, voltage, electrochemical, and cancer therapy.

\section{INTRODUCTION}

Cancer is a disease caused by the uncontrolled and massive growth of abnormal cells. It has several types, one of which is blood or hematologic cancer. There are three main types of blood cancer, namely Leukemia, Lymphoma Myeloma, and Plasma Cell Disorders (Rodriguez-Abreu et al., 2007). Leukemia is a metastatic and malignant disease of the bloodmaking organs (bone marrow) caused by preferences and problems with the proliferation of white blood cells which happen in the blood (partial blood cells in the bone marrow are called blasts). Patients with Leukemia have high and uncontrolled production of blood. Data from 2013-2017, showed that the rate of new leukemia cases was 14.1 per 100,000 men and women in a year, with a death rate of 6.3 per 100,000 men and women a year. Presently, the common methods used in leukemia treatment are chemotherapy, radiotherapy, and stem cell transplant which have side effects and are costly. However, an alternative, Electro Capacitive Cancer Treatment (ECCT) was developed by Warsito (2012) and is currently developing cancer therapy using an electric field. The ECCT method uses an alternating electric field which penetrates the cell membrane and affect the splitting cells, thereby killing and inhibiting cancer cell growth (Palti, 2007). These electric fields from the alternating source require polar molecules to oscillate, leading to a lower effect as frequency increases (Kirson et al., 2007). Furthermore, AC electric fields with frequencies above $100 \mathrm{kHz}$ are known to have no significant biological effects (Kirson et al., 2004).

The homogenous electric fields direct their dipole molecules along the lines of force (Zhao et al., 1999), while inhomogeneous fields direct dipole molecules toward the region of high field intensity, and this is called positive dielectrophoresis (Ma et al., 2011). Conversely, negative dielectrophoresis occurs when particles move to the weak part of an electric field (Doh and Cho, 2005). White blood cell division in leukemia patients occurs very quickly, therefore the number of blasts in 
the blood is high. During mitosis, cells tend to be polar (Pasquier and Kavallaris, 2007), and the force exerted by electric fields on polar molecules is maximum when the dipole orientation is in the direction of the fields (Bethlem, 2002).

Alamsyah et al., (2015) demonstrated antiproliferative effects of electric field on the cancer cell and based on Justin et al., (2011) voltage breaks down leucocyte into electric current. Furthermore, according to Hiroko et al., (2013), it separated leukemia cells from the blood and assist migration to the region of high voltage. Using both concepts above, the arrangement of the electrodes in an electric field distribution was studied, as a potential blood cancer therapy. The Electric field distribution was computed by COMSOL Multiphysics software. The software solved Partial Differential Equation (PDE) associated with physical law via the Finite Element Method (FEM), which was used on complex geometries and handles discontinuous gradient of variables. Furthermore, numerical errors were resolved by varying or refining the mesh in that area (Dickson et al., 2014).

The arrangement of the electrodes determines the distribution of the electric field in the blood. Khorsoej et al., (2018), subsequently investigated electrode position for electric field distribution in the human brain, showed results of two optimal electrode arrangements that are orthogonal to each other. Furthermore, Andiani et al., (2017) carried out a study of electric field distribution for blood cancer using a voltage of $10 \mathrm{~V}$ and frequencies of $100 \mathrm{kHz}$ and $200 \mathrm{kHz}$, which lead to a change in the electric field distribution in the presence of blood cancer.

Three model arrangements of electrodes to determine the most optimal electric field distribution were proposed. In this case, leukemia was studied using parameters of normal and abnormal blood as a medium. The objective of the study was to obtain the optimal electrode arrangement for use in blood cancer therapy.

\section{METHODS}

\section{Model}

The modeling stage consists of the determination of the design parameters and geometry. In this study, the geometry of the electrode is made of conductive fabric with $\mathrm{Ag}$ deposits which becomes an Ag nanorod with a radius of $0.2 \mu \mathrm{m}$ and $1 \mu \mathrm{m}$ in height on the conductive fabric substrate. However, the blood geometry is made rectangular thereby simplifying the model to geometry of $60 \mathrm{~mm} \times$ $60 \mathrm{~mm} \times 90 \mathrm{~mm}$. All design geometries were created by COMSOL Multiphysics 5.4. The electrode design is shown in Figure 1.

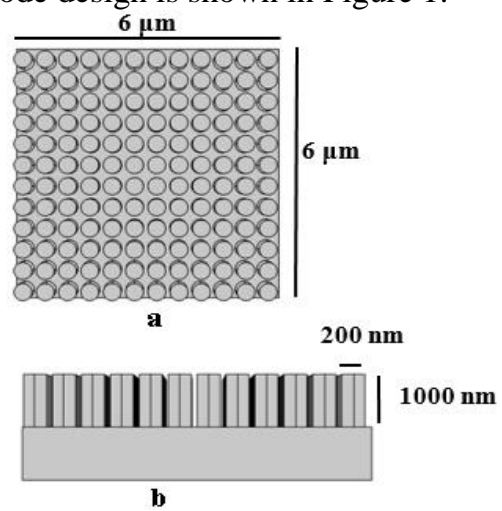

Figure 1. Electrode design a) top view, b) side view
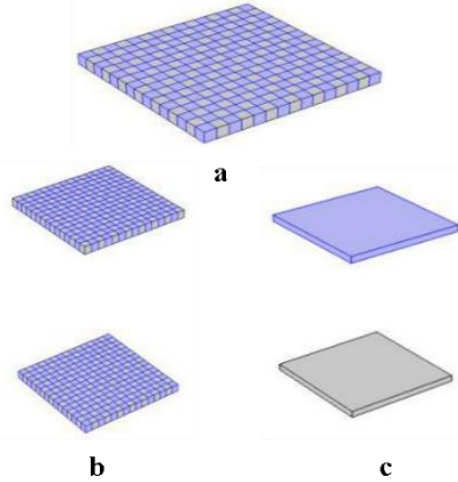

Figure 2. Electrode arrangement, a) model 1, b) model 2, c) model 3 (blue positive potential and gray is negative potential).

\section{Simulation}

The simulation stage was carried out using the electrostatic theoretical approach. Here, the physical parameter settings were executed using COMSOL Multiphysics 5.4 and they include subdomain, boundary, free mesh parameter and solver parameter. A scheme of this stage can be seen on the flowchart in Figure 3. The subdomain stage involves the setting of the type of material and physical parameters. An example of the physical parameter used is relative permittivity $\left(\varepsilon_{\mathrm{r}}\right)$ as summarized in Table 1 . The region of simulation on the object and electrode is limited using a boundary and for the free mesh parameter, used the finer size was used. In the solver stage set simulation parameters related 
to physics and study parameters were used. The physics parameter (electrostatics) obtained the distribution of the electric field and frequency domain method during the study stage.

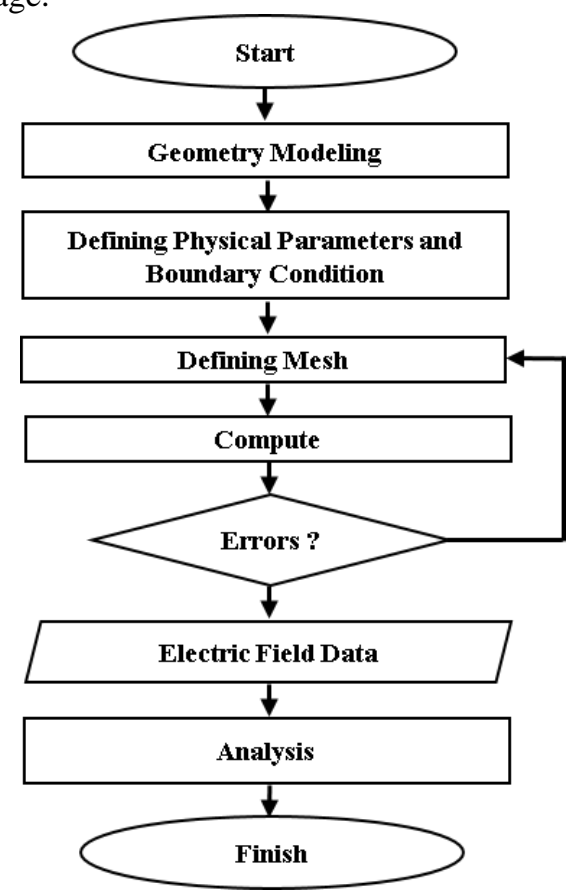

Figure 3. Flowchart based on how to develop simulation analysis for the effect of electric field on blood cancer.

There are 3 types of the electrode arrangement. Model 1 is the type in which plates were placed on one side of the object and the electrode arrangement had electric poles in pairs. For model 2, electrode plates were placed on two sides of the object and the electrode arrangement had pairs of electric poles. Finally, in model 3 plates were placed on two sides of the object and electrode poles were opposite to each other. The illustration of the model is shown in Figure 2.

Table 1. Physical parameters for material subdomain setting (IFAC, 2020) (Surowiec et al., 1985).

\begin{tabular}{ccc}
\hline \multirow{2}{*}{ Material } & \multicolumn{2}{c}{ Relative Permittivity } \\
\cline { 2 - 3 } & $100 \mathrm{kHz}$ & $200 \mathrm{kHz}$ \\
\hline Air & 1 & 1 \\
Silver (Ag) & 1 & 1 \\
Normal Blood & 5120 & 4930 \\
Lymphocytes B & $5063^{*}$ & - \\
Lymphocytes T & $4650 *$ & - \\
\hline
\end{tabular}

* Lymphocytes B and T measured on volume 0.63; $0.41\left(\mu \mathrm{L}^{-1}\right)$ and frequency $20 \mathrm{kHz}$.

\section{RESULTS AND DISCUSSION}

The first analysis in this study was the electric field data from each mesh point which was plotted in $3 \mathrm{D}$ to see the field distribution in the object volume. Furthermore, the analysis determined how well the distribution of the object of the electric field was for each arrangement of the electrodes. The second analysis subsequently makes use of a straight line plotted along the $\mathrm{Z}$-axis from center of the $\mathrm{X}-\mathrm{Y}$ plane, where the electric field data along the straight line is plotted in a one-dimensional graph of the electric field $(\mathrm{V} / \mathrm{m})$ relationship with $\mathrm{Z}$ coordinates ( $\mathrm{mm}$ ).

Studies with variation in the location of electrodes were carried out for the comparison of electric field distributions generated by electrodes on objects with voltage, medium and electrode arrangement variations (model 1, model 2 and model 3). The first study was carried out on the electrode setup variations on the air medium with an input voltage of $0.34 \mathrm{~V}$ and a frequency of $100 \mathrm{kHz}$ to determine the optimal spread of the electric field. There were 3 model setups carried out. The result of the distribution of electric fields in an air medium with variations in electrode arrangement is shown in Figure 4. Furthermore, It appeared that the field distribution in the setup with model 1 and model 2 had a good distribution on the surface around the electrode alone, however it had a poor depth of the object characterized by, the small value of the field inside the object as indicated by the magnitude of the electric field near the electrode compared to the distance from the electrode.

The plot of the electric field along the Zaxis is shown in Figure 5. The magnitude of the electric field near electrode inside model 1 and model 2 are $25.04 \mathrm{~V} / \mathrm{m} ; 3.13 \times 10^{-4} \mathrm{~V} / \mathrm{m}$ and $27.4 \mathrm{~V} / \mathrm{m} ; \quad 2.8 \times 10^{-3} \mathrm{~V} / \mathrm{m}$ respectively. However, the electrode setup of model 3 has better distribution than the setup of model 1 and model 2. This is characterized by the magnitude of electric field near the electrode inside the object. Furthermore, the difference in magnitude was not too large, since the magnitude of the electric field near the electrode and inside the object was $22.4 \mathrm{~V} / \mathrm{m}$ and $11.4 \mathrm{~V} / \mathrm{m}$ respectively. Therefore, by structuring the electrode it is expected that model 3 provides good field distribution in abnormal blood and increase efficacy in blood cancer therapy. 


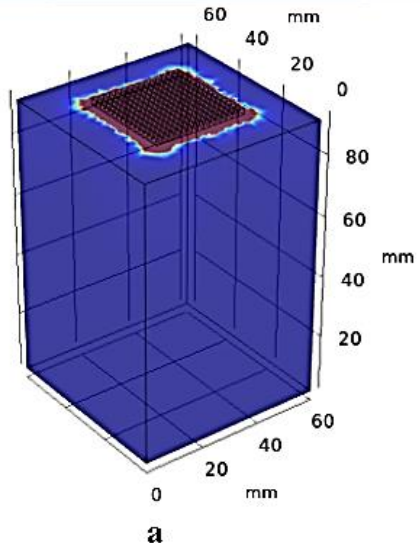

a
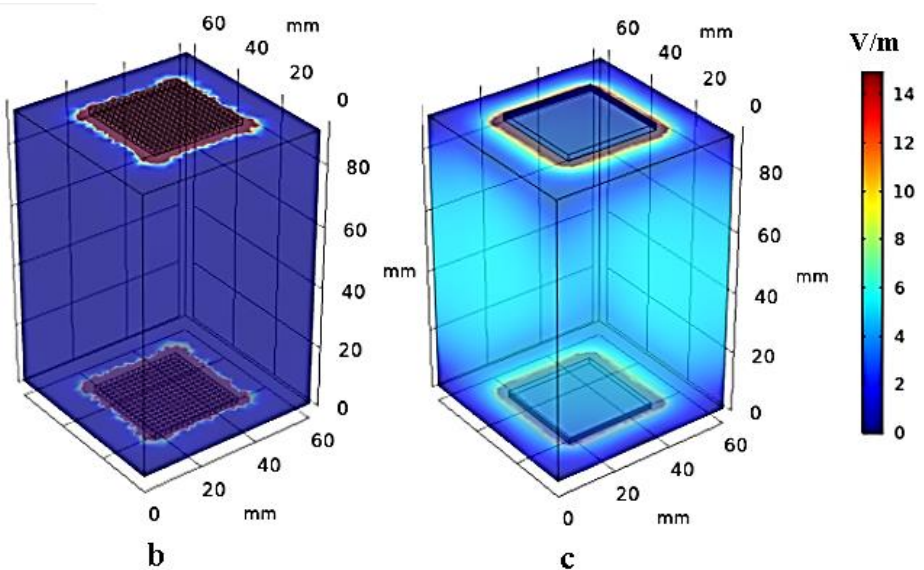

Figure 4. Result of the electric field distribution on air as a material type a) model 1, b) model 2, and c). model 3 , respectively.

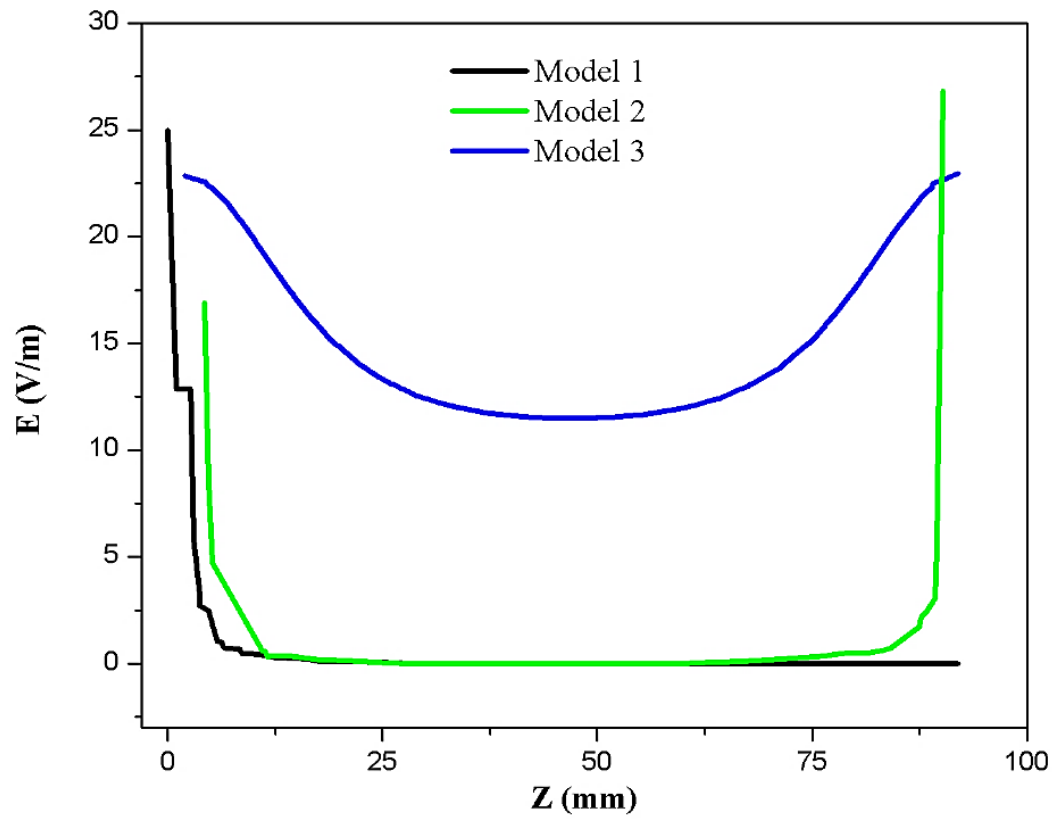

Figure 5. The curve of the electric field distribution along the Z-axis at the center of the X-Y plane in air medium and the input voltage $0.34 \mathrm{~V}$, with three variations of the electrode arrangement.

Based on the results of the arrangement of model 3, electrodes had better distribution than model 1 and model 2. The medium was therefore replaced with normal blood, lymphocyte $\mathrm{B}$ and lymphocyte $\mathrm{T}$, which in this study physical parameters of lymphocytes at frequencies of $20 \mathrm{kHz}$ were used by Surowiect et al., (1985). In addition to the limitations of relative permittivity data of lymphocytes from
Surowiect et al., (1985), showed a decrease in the relative permittivity value due to increased frequency was not too significant within the frequency range of $20 \mathrm{kHz}$ to $200 \mathrm{kHz}$, for both lymphocyte B and lymphocyte T. Therefore, the relative permittivity data of $20 \mathrm{kHz}$ frequency remains and frequencies of $100 \mathrm{kHz}$ and $200 \mathrm{kHz}$ were used in this study. 


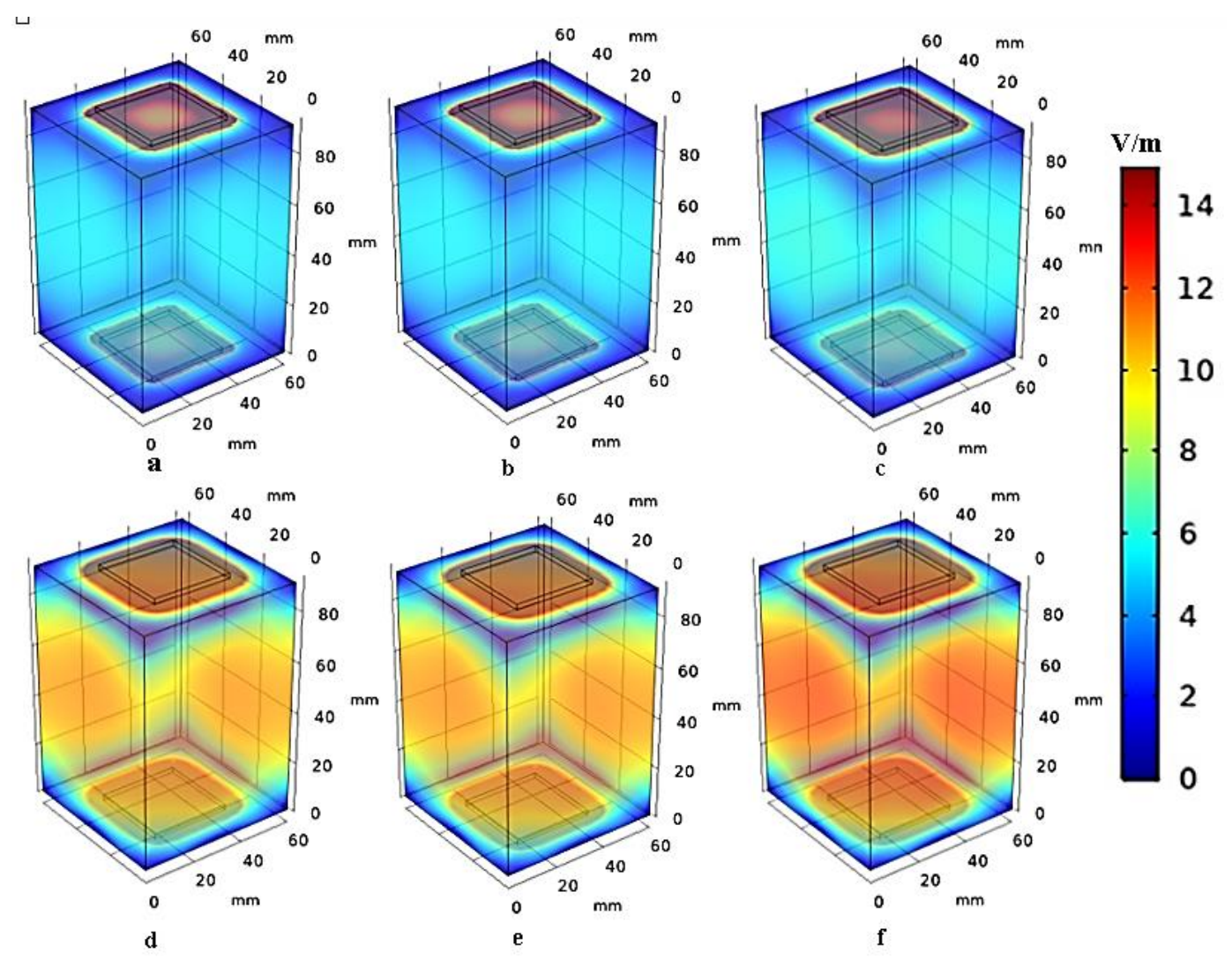

Figure 6. The simulation results of the electric field distribution with the electrode arrangement model 3 in blood medium a) normal blood $0.34 \mathrm{~V}$, b) lymphocytes $\mathrm{B} 0.34 \mathrm{~V}$, c) lymphocytes $\mathrm{T} 0.34 \mathrm{~V}$, d) normal blood $0.6 \mathrm{~b} \mathrm{~V}$, e) lymphocytes $\mathrm{B} 0.68 \mathrm{~V}$ and f) lymphocytes $\mathrm{T} 0.68 \mathrm{~V}$.

Figure 6 shows the distribution of electric field in normal blood (a) lymphocyte B (b) and lymphocyte $\mathrm{T}$ (c) with input voltage $0.34 \mathrm{~V}$, and (d) normal blood, (e) lymphocyte B (f) lymphocyte $\mathrm{T}$ with input voltage $0.68 \mathrm{~V}$. The distribution of electric fields from the three different objects with input voltage $0.34 \mathrm{~V}$ did not show significant change, due to the permittivity value of the materials being approximately equal. Subsequently, doubling of the input voltage led to results appearing to have a slight difference from the distribution of electric field from the three mediums, as seen in Figure 6 parts (d), (e) and (f). This showed that the normal blood had an electric field magnitude smaller than lymphocyte $\mathrm{T}$, characterized by the darker color of the $3 \mathrm{D}$ plot of the lymphocyte $\mathrm{T}$ object.

The distribution of the electric field with input voltages of $0.34 \mathrm{~V}$ and $0.68 \mathrm{~V}$ are plotted along the Z-axis at the center of the X-Y plane, seen in Figure 7. However, at an input voltage of $0.34 \mathrm{~V}$, the three mediums appeared to have coinciding electric field distribution. Meanwhile, at input voltage $0.68 \mathrm{~V}$, there is a slight gap between lymphocyte $\mathrm{T}$ medium and normal blood. Consequently, input voltage of $0.34 \mathrm{~V}$ for lymphocyte $\mathrm{T}$ had a field magnitude greater than normal blood and lymphocyte $\mathrm{B}$, had maximum values at $\mathrm{Z}=2 \mathrm{~mm}$ and $\mathrm{Z}=92$ $\mathrm{mm}$, with values of $12.1 \mathrm{~V} / \mathrm{m}$ and $12.1 \mathrm{~V} / \mathrm{m}$, respectively. However, the lowest field distribution values were in normal blood with $100 \mathrm{kHz}$ frequency and a maximum value at point $\mathrm{Z}=2 \mathrm{~mm}$ and $\mathrm{Z}=90 \mathrm{~mm}$, having 11.42 $\mathrm{V} / \mathrm{m}$ and $11.48 \mathrm{~V} / \mathrm{m}$ respectively. At the same point, for a frequency of $200 \mathrm{kHz}$ maximum values of $11.86 \mathrm{~V} / \mathrm{m}$ and $11.92 \mathrm{~V} / \mathrm{m}$ were obtained, and for lymphocyte $\mathrm{B}$ with electric field values of $11.5 \mathrm{~V} / \mathrm{m}$ and $11.61 \mathrm{~V} / \mathrm{mm}$. 


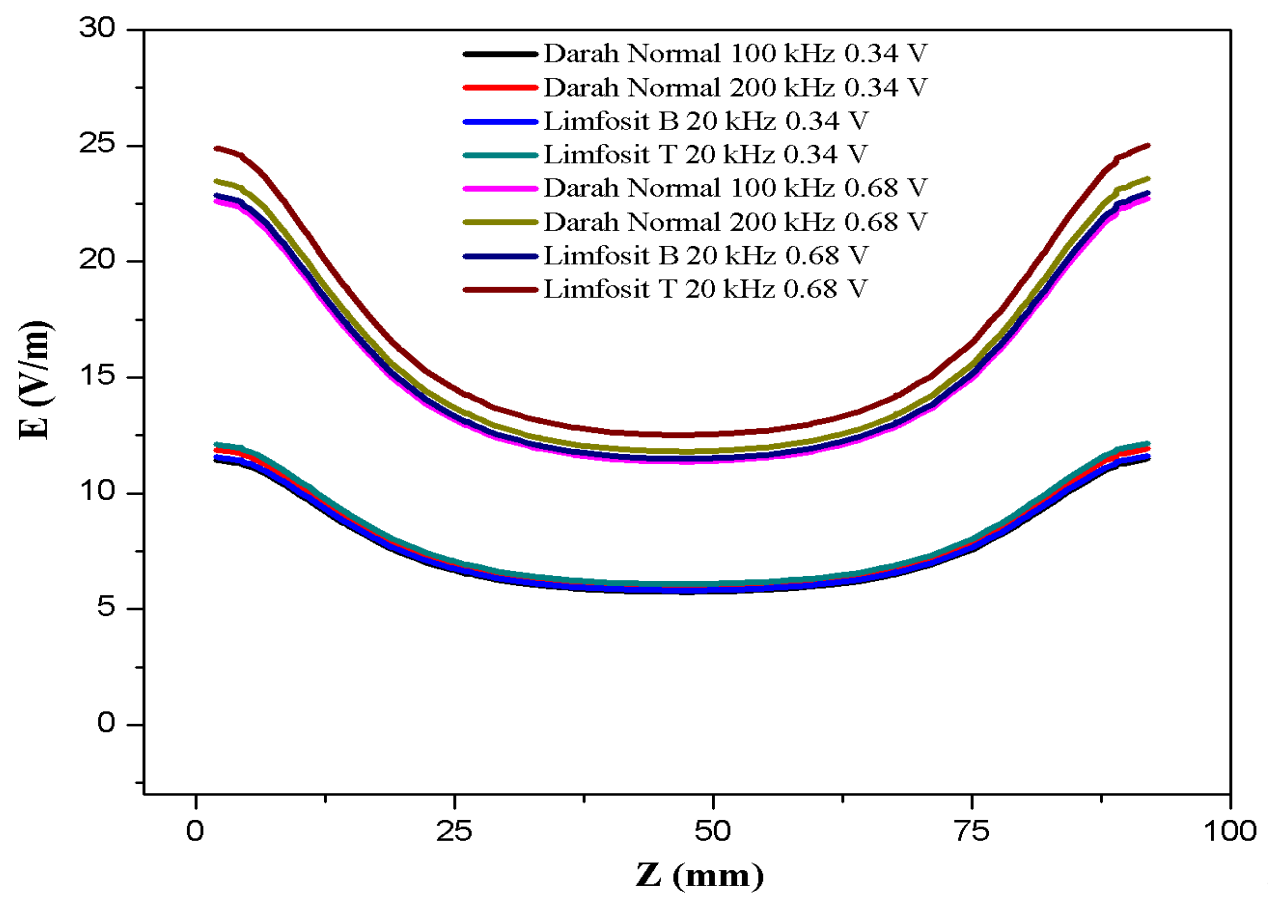

Figure 7. The curve of the electric field distribution along Z-axis at the center of $\mathrm{X}-\mathrm{Y}$ plane in blood medium with variation of the input voltage is $0.34 \mathrm{~V}$ and $0.68 \mathrm{~V}$.

The distribution of the electric field for an input voltage of $0.68 \mathrm{~V}$ is similar to one having an input voltage of $0.34 \mathrm{~V}$. However, the value of the electric field is greater than that having an input voltage of $0.34 \mathrm{~V}$ at point $\mathrm{Z}=2 \mathrm{~mm}$ and $\mathrm{Z}=92 \mathrm{~mm}$ for $100 \mathrm{kHz}$ and $200 \mathrm{kHz}$. When normal blood lymphocyte $\mathrm{B}$ and lymphocyte $\mathrm{T}$ had maximum values of 22.6 $\mathrm{V} / \mathrm{m} ; 22.7 \mathrm{~V} / \mathrm{m}, 23.47 \mathrm{~V} / \mathrm{m} ; 23.59 \mathrm{~V} / \mathrm{m}, 22.85$ $\mathrm{V} / \mathrm{m} ; 22.97 \mathrm{~V} / \mathrm{m}$ and $24.88 \mathrm{~V} / \mathrm{m} ; 25.01 \mathrm{~V} / \mathrm{m}$. Therefore, increasing the input voltage increase the magnitude of the electric field in the medium and results obtained from the CNT dielectrophoretic simulation, showed the direct proportionality of voltage to the magnitude of the electric field (Uchechukwu et al., 2012).

The maximum value of the electric field for all objects is located near the electrode, as justified in this study, since the negative gradient potential will be directly proportional to the electric field. Furthermore, the electric field at points near the voltage source will have large strengths compared to the two electrodes, seen in Figure 7. The minimum value occurs at coordinate $Z=40 \mathrm{~mm}$ and $Z=60 \mathrm{~mm}$, because it is the point furthest from both electrodes when comparing a given minimum voltage. Another factor affecting the distribution of the electric field is electrode permittivity, when the relative permittivity of the electrode is greater than the liquid, the field will be larger at the near electrode (especially at the point of contact) and will be weaker in the gaps of both electrodes (Morgane et al., 2015). Conductive fabric electrodes that have been repositioned with $\mathrm{Ag}$, to obtain a permittivity value of conductive material were infinite and this was used in this study. However, the permittivity value entered as 1, making it correct for the greatest electric field to be present near electrodes or their contact points.

Dielectrophoretic force (DEP) is a phenomenon experienced by particles in a suspension subjected to an inhomogeneous electric field (usually generated by AC signals) (Becker et al., 1994). Subsequently, the force decreases exponentially for electrodes (Pethig and Markx, 1997) at low frequencies depending on conductivity, however, at high frequencies there is much influence by the permittivity. There is a consequent increase in the force when the permittivity is greater than the surrounding medium, therefore particles migrate to a larger electric field associate with positive dielectrophoresis. Conversely, when the permittivity of the particles is smaller than the medium, the particles will migrate to a weak electric field associated with negative dielectrophoresis (Huang and Pethig, 1991). Therefore, from these studies carried out, at 
frequencies of $100 \mathrm{kHz}$ and $200 \mathrm{kHz}$, the permittivity of lymphocyte $B$ and lymphocyte $\mathrm{T}$ is less than the blood, which is considered the medium, leading to negative dielectrophoresis, where lymphocyte B and lymphocyte $\mathrm{T}$ will move towards weak electric field areas. Consequently, the breakdown of leukocytes with input voltage is difficult to carry out.

In comparison, the addition of photosensitizer (PS) in the blood is one way to lower the value of blood permittivity. Furthermore, Porphyrin data with a permittivity value of 6.78 (Mota et al., 2017) was obtained from the injection of blood with a volume ratio is 90:10. The equation for calculating the dielectric of the combination of solutions carried out by Jouyban et al., (2004) is.

$$
\varepsilon_{m}=\varepsilon_{1} \phi_{1}+\varepsilon_{2} \phi_{2}
$$

Where $\varepsilon_{1}, \varepsilon_{2}$ is the permittivities of solutions 1 and 2 , with $\phi_{1,} \phi_{2}$ as the volumes (moles, mass and other) of solutions 1 and 2. By using the equation, the value of permittivity from blood decreased from 5120 to 4353. Therefore, with the addition of PS permittivity of the blood medium was lowered to make lymphocyte particles experience positive DEP and move towards areas with large electric fields. Furthermore, the addition of Porphyrin to the blood in electric field study is to increase lysis cell in the blood, therefore blast cells are able to break down (Ward et al., 1996).

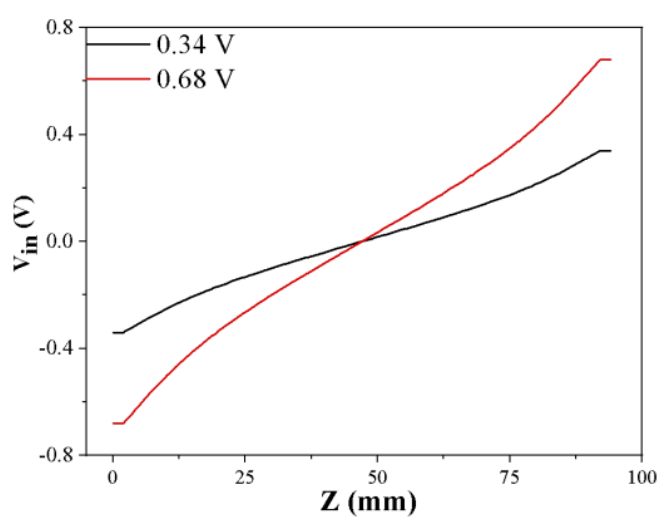

Figure 8 . The curve of the voltage along the Zaxis in the center of $\mathrm{X}-\mathrm{Y}$ plane with input voltage $0.34 \mathrm{~V}$ and $0.68 \mathrm{~V}$.

The area near the electrode also had an input voltage when the particles were in a large area of the electric field. Subsequently, this voltage is expected to aid the breakdown of leukocyte cells into electron currents. Justin et al., (2011) carried out a study related to the conversion of leukocytes into electric currents.
The results of the study showed that a minimum voltage of $0.34 \mathrm{~V}$ was needed to break down leukocytes into electric currents and enable the oxidization of leukocyte cells in blood cancer. An increase in the input voltage value twice the initial $(0.68 \mathrm{~V})$ was used to view which area had voltage worth $0.34 \mathrm{~V}$. A graph of voltage distribution on the object is shown in Figure 8. It appears that the voltage Of $0.34 \mathrm{~V}$ only occurs at a distance of $\mathrm{Z}=2$ $\mathrm{mm}$ and $\mathrm{Z}=90 \mathrm{~mm}$ at the surface of the electrode. Finally, increasing the voltage, at point $Z=19.6 \mathrm{~mm}$ and $Z=75.1 \mathrm{~mm}$ will cause the minimum voltage reached to break leukocyte cells into electric currents. Through increased voltage, the incidence of leukocyte rupturing occurs when electric currents increase.

\section{CONCLUSION}

The conclusion from the studies carried out is that the electrode arrangement producing an even distribution of electric field is used in model 3. Furthermore, for the conversion of leukocytes into electric current due to the input voltage, stimulation of blood cancer therapy through a dielectrophoretic mechanism model was used. PS Porphyrin was added to the blood causing a decrease in its permittivity value to 4353, therefore, stimulating positive dielectrophoretic phenomena. This leads to the migration of leukocyte cells to an area with a strong electric field, and sufficient input voltage value to convert the leukocyte cells into electric current. In future studies, the addition of electrodes can be carried out to increase the intensity of the electric field and specific blood absorption rate, including detailed studies related to the movement of blood as they approach the electrode due to the dielectrophoretic effect.

\section{ACKNOWLEDGMENT}

This work was supported by the Institut Teknologi Sepuluh Nopember (ITS) and RISTEK-BRIN under Penelitian Dasar (PD) (No. 1107/PKS/ITS/2020)

\section{REFERENCE}

Alamsyah F, I.N. Ajrina, F.N.A. Dewi, D. Iskandriati, S.A. Prabandari, and W.P. Taruno. 2015. "Antiproliferative Effect of Electric Fields on Breast Tumor Cells In Vitro and In Vivo." Indonesian Journal of Cancer Chemoprevention. 6(3): 71-77. 
Andiani L, Endarko, Mahfudz Al Huda, and W.P. Taruno. 2017. "A Novel Method For Analyzing Electric Field Distribution Of Electro Capacitive Cancer Treatment (Ecct) Using Wire Mesh Electrodes: A Case Study Of Brain Cancer Therapy." Euromediterranean Biomedical Journal. 12(38): 178-183.

Beckert F. F., X-B Wangt, Y Huangt, R Pethigt, J Vykoukalt and P R C Gascoyne. 1994. "The removal of human leukaemia cells from blood using interdigitated microelectrodes." J. Phys. D: Appl. Phys 27: 2659-2662.

Bethlem, H.L. 2002. Deceleration and Trapping of Polar Molecules Using Timevarying Electric Fields. Netherlands: Thesis. Katholieke Universiteit Nijmegen.

Dickinson, E.J.F., H. Ekström and E. Fontes. 2014. "COMSOL Multiphysics ®: Finite element software for electrochemical analysis. A mini review." Electrochem. Commun. 40: 71-74.

Doh, I. and Y.H. Cho. 2005. "A continuous cell separation chip using hydrodynamic dielectro-phoresis (DEP) process." Sens. Actuators A: Phys. 121(1): 59-65.

Hiroko, I., T. Yamakawa and M. Eguchi. 2012. "Separation of Leukemia Cells from Blood by Employing Dielectrophoresis." Intelligent Automation \& Soft Computing 18(2): 139-152.

Huang Y. and R. Pethig. 1991. "Elecrrode Design for Negative Dielectrophoresis." Measurement Science and Technology. 2(12): 1142-1146.

IFAC. 2020. Calculation of the Dielectric Properties of Body Tissues in the frequency range $10 \mathrm{~Hz}-100 \mathrm{GHz}$. Agustus 31.

Justin, G. A, Y. Zhang, X. T. Cui, C. W Bradberry, M. Sun and R. J. Sclabassi. 2011. "A Metabolic Biofuel Cell: Conversion of Human Leukocyte Metabolic Activity to Electrical Currents." Journal of Biological Engineering. 5(5).

Jouyban A., S. Soltanpour and H.K. Chan. 2004. "A simple relationship between dielectric constant of mixed solvents with solvent composition and temperature." International Journal of Pharmaceutics 269: 353-360.

Kirson, E.D, V. Dbalý, F. Tovarys, J. Vymazal, J.F. Soustiel, A. Itzhaki, D. Mordechovich, S. Steinberg-Shapira, Z. Gurvich, R.
Schneiderman, Y. Wasserman, M. Salzberg, B. Ryffel, D. Goldsher, E. Dekel and Y. Palti. 2007. "Alternating electric fields arrest cell proliferation in animal tumor models and human brain tumors." Proc Natl Acad Sci U S A. 104(24): 10152101527.

Kirson E.D, Z. Gurvich, R. Schneiderman, E. Dekel, A. Itzhaki, Y. Wasserman, R. Schatzberger and Y. Palti. 2004. "Disruption of cancer cell replication by alternating electric fields." Cancer Res. 64(9): 3288-3295.

Korshoej A.R, F.L. Hansen, N. Mikic, G. von Oettingen, J.C.H. Sørensen and A. Thielscher. 2018. "Importance of electrode position for the distribution of tumor treating fields (TTFields) in a human brain. Identification of effective layouts through systematic analysis of array positions for multiple tumor locations." PLoSONE. 13(8): e0201957.

Ma, W., T. Shi, Z. Tang, S. Liu, R. Malik and L. Zhang. 2011. "High-throughput Dielectrophoretic Manipulation of Bioparticles Within Fluids Through Biocompatible Three Dimensional Microelectrode Array." Electrophoresis. 32(5): 494-505.

Morgane C, O. Larue and E. Vorobiev. 2015. "Electric (Electro/ Dielectro-Phoretic) dForce Field Assisted Separators." In Progress in Filtration and Separation, by Steve Tarleton, 325-397. London: Elsevier.

Mota J.P. F., A. E. da Costa Júnior, V. G. P. Ribeiro, S. G. Sampaio, N. M. A. Lima, F. L. F. da Silva, C. S. Clemente, G. Mele, D. Lomonaco and S. E. Mazzetto. 2017. "Synthesis, Characterization and Dielectric Properties of New 5-(4-Hydroxyphenyl)10,15,20-tri-4-[2-(3entadecylphenoxy)ethoxy]phenyl porphyrin and Their $\mathrm{Ni}, \mathrm{Co}$ and $\mathrm{Cu}$ Complexes." J. Braz. Chem. Soc. 28(6): 1063-1073.

Palti. 2007. Method for Selectively Destroying Dividing Cells. Patent Application Publication. United States Patent 20070033660A1. February 8.

Pasquier, E. and M. Kavallaris, 2007. "Microtubules: a Moving Target in Cancer Therapy." IUBMB lIFE. 60(3): 165-70.

Pethig, R. and G. H. Markx. 1997. "Applications of dielectrophoretic in 
biotechnology." Trends Biotechnol. 15: Uchechukwu C. Wejinya, N. Xi, and K. Wai C. 426-432.

Rodriguez-Abreu D, A. Bordoni and E. Zucca. 2007. "Epidemiology of hematological malignancies." Annals of Oncology. 18(3): 13-18.

SEER. 2020. SEER Stat Fact Sheets: Leukemia - Cancer Stat Facts. Agustus 30. https://seer.cancer.gov/statfacts/html/leuks. html.

Surowiec A., S. S. Stuchly and C. Izaguirre. 1986. "Dielectric properties of human B and $\mathrm{T}$ lymphocytes at frequencies from 20 kHz to 100 MHz." Phys. Med. Biol. 31(1): 43-53.

Lai. 2012. "Design and Generation of Dielectrophoretic Forces for Manipulating Carbon Nanotubes." NanoOptoelectronic Sensors and Devices. 2949.

Ward T., A. Rollan, G. Flynn, and A.P. McHalea. 1996. "The effects of electric fields on photosensitized erythrocytes: possible enhancement of photodynamic activation." Cancer Letters. 106: 69-74.

Zhao, M., Forrester, J.V. and McCaig, C.D. 1999. "A Small, Physiological Electric Field Orients Cell Division." Proc. Natl. Acad. Sci. USA. 96(9): 4942-4946.

Taruno, W. P. 2012. Indonesia Patent REG P00201200092. 
